\title{
Experimental Organism Benign Nephroblastoma Neoplasm
}

National Cancer Institute

\section{Source}

National Cancer Institute. Experimental Organism Benign Nephroblastoma Neoplasm. NCl Thesaurus. Code C114235.

A benign embryonal neoplasm of the kidney. 